\title{
Measurement of the structure function $F_{L}$ at HERA
}

\author{
S. Glazov ${ }^{\mathrm{a}}$ (on behalf of the H1 and ZEUS collaborations) \\ ${ }^{\mathrm{a} D E S Y}$ \\ Notkestrasse 85, \\ 22607 Hamburg, Germany
}

Measurements of the structure function $F_{L}$ performed at the HERA collider are reported. The results are based on the neutral current inclusive $e^{+} p$ scattering cross section measured with $e^{+} p$ data collected at a positron beam energy of $27.6 \mathrm{GeV}$ and proton beam energies at 460,575 and $920 \mathrm{GeV}$. The measured structure function $F_{L}$ is consistent with QCD expectations.

\section{Introduction}

Deep inelastic lepton-hadron scattering (DIS) plays an important role for the understanding of the structure of the nucleon and of the dynamics of parton interactions. The discovery of Bjorken scaling [1] and its violation [2] at fixed target experiments, first initiated development of the parton model and then of the theory of strong interactions, Quantum Chromodynamics (QCD).

At low $Q^{2}$, the inclusive double differential $e p$ scattering cross section in a reduced form can be represented by two structure functions:

$$
\begin{aligned}
\sigma_{r}\left(x, Q^{2}\right) & =F_{2}\left(x, Q^{2}\right)-f(y) F_{L}\left(x, Q^{2}\right), \\
f(y) & =\frac{y^{2}}{Y_{+}}, \quad Y_{+}=1+(1-y)^{2} .
\end{aligned}
$$

Here $Q^{2}$ is the absolute four momentum transfer squared, $x$ is the Bjorken scaling variable, and the inelasticity $y$ is related with $x, Q^{2}$ and the centre-of-mass energy squared $S$ as $y=Q^{2} /(S x)$. The structure functions $F_{2}$ and $F_{L}$ correspond to the scattering cross sections for transversely and longitudinally polarised photons: $F_{2} \sim \sigma_{T}+\sigma_{L}$ and $F_{L} \sim \sigma_{L}$. Due to the kinematic factor $f(y)$ and the relation $0 \leq F_{L} \leq F_{2}$, the $F_{L}$ term has a numerically small influence on the cross section for $y<0.6$.

In the quark parton model for massless spin $1 / 2$ quarks $\sigma_{L}=0$ is predicted from angular momentum conservation. The observation that $F_{L}$ indeed is small at fixed target experiments [3] thus played an important role for the formation of the partonic picture. In NLO DGLAP [4], gluon emission lifts the angular momentum restriction and $F_{L}$ acquires a non-zero value. Measuring $F_{L}$ thus allows for the determination of the gluon density using the cross section helicity decomposition.

The experimentally accessible kinematic range of DIS has been vastly extended since the startup of the HERA $e p$ collider due to its high centreof-mass energy. Operating at the nominal lepton and proton beam energy of $E_{e}=27.6 \mathrm{GeV}$ and $E_{p}=920 \mathrm{GeV}$, respectively, $x$ values of $\approx 10^{-4}$ can be reached at $Q^{2} \approx 10 \mathrm{GeV}^{2}$. Since the bulk of the measurements at the nominal centreof-mass energy corresponds to $y<0.6$, the cross section data can be interpreted as a measurement of the structure function $F_{2}$. For small $x$ the proton structure is dominated by the sea and gluon quark densities, which in DGLAP can be determined via $F_{2}$ and $\mathrm{d} F_{2} / \mathrm{d} \ln Q^{2}$.

Although the accuracy of the combined HERA experimental data allows a precise determination of the sea and gluon density [5], questions may be raised about validity of the DGLAP approach at low $x$ in which $\alpha_{S} \ln Q^{2}$ terms are summed while contributions of $\alpha_{s} \ln (1 / x)$ terms become large as well. The parton dynamics at low $x$ may be better approximated by different evolution equations, such as BFKL [6], CCFM [7] or other non-linear equations [8]. These different evolution schemes may affect the structure functions $F_{2}$ and $F_{L}$ differently; for a summary see for example [9]. A 


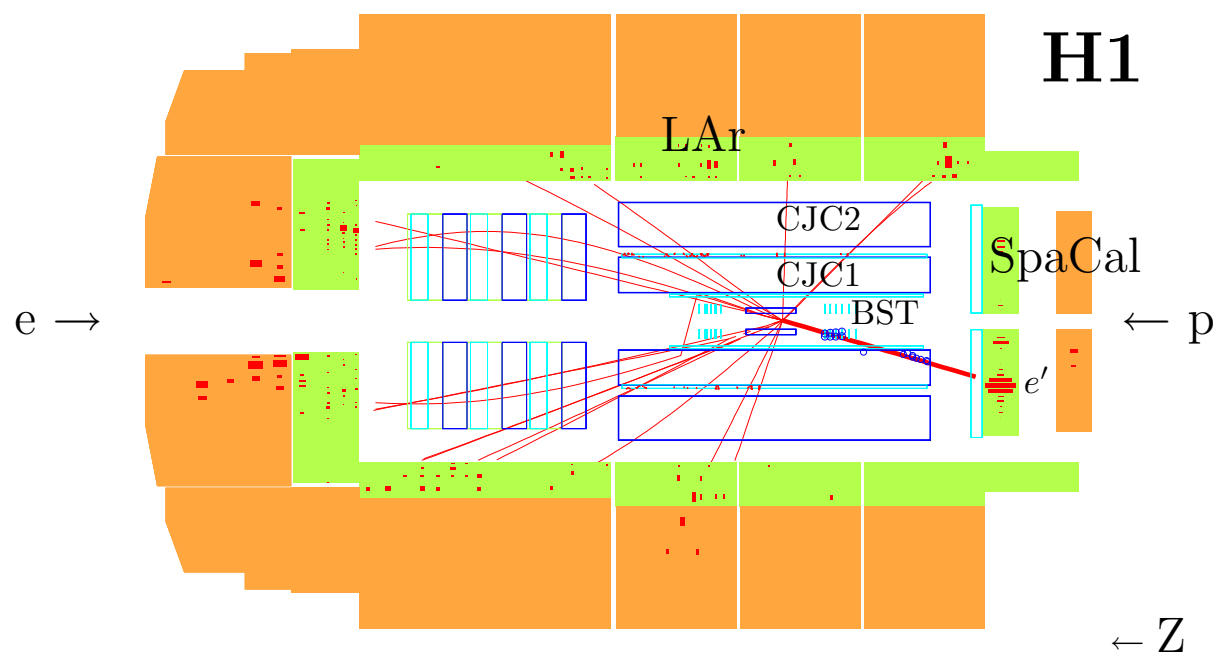

Figure 1. A view of a high $y$ event reconstructed in the $\mathrm{H} 1$ detector. The electron and proton beam directions are indicated by the arrows. For the coordinate system used at HERA the $z$ axis points in the direction of the proton beam. The interaction vertex is reconstructed using the hadronic final state (thin lines) and the scattered electron (thick line) tracks in the central tracker. The central tracker consists of (from the beam line outwards) the silicon tracker and the drift chambers CJC1 and CJC2 and is surrounded by the liquid argon (LAr) calorimeter. The scattered electron trajectory is reconstructed in the backward silicon tracker BST and the CJC1. The electron energy is measured in the electromagnetic part of the SpaCal calorimeter.

measurement of the structure function $F_{L}$ thus provides an important cross check of the theory.

To determine the two structure functions $F_{2}\left(x, Q^{2}\right)$ and $F_{L}\left(x, Q^{2}\right)$ from the reduced cross section, equation 1 , it is necessary to perform measurements at the same values of $x$ and $Q^{2}$ but different $y$. This is achieved at HERA by reducing the proton beam energy. Two runs at reduced proton beam energy $E_{p}=460 \mathrm{GeV}$ and $E_{p}=575 \mathrm{GeV}$ were performed with an integrated luminosity of about 13 and $6 \mathrm{pb}^{-1}$, respectively. The run at $E_{p}=460 \mathrm{GeV}$ gives highest sensitivity to $F_{L}$ while the run at $E_{p}=575 \mathrm{GeV}$ extends the kinematic range of the measurement and provides an important cross check. Using these data, the measurements of the reduced cross section and $F_{L}$ are performed by the $\mathrm{H} 1$ and ZEUS collaborations. An event at high inelasticity, reconstructed in the $\mathrm{H} 1$ detector, is shown in figure 1.

\section{Cross section measurement at high $y$}

The inclusive cross section is sensitive to $F_{L}$ only at large $y$. In this region, the event kinematics is best reconstructed using the so-called electron method in which $Q^{2}, y$ and $x$ are reconstructed using the scattered electron energy $E_{e}^{\prime}$ and the scattering angle $\theta_{e}$ :

$Q^{2}=\frac{E_{e}^{\prime 2} \sin ^{2} \theta_{e}}{1-y}, \quad y=1-\frac{E_{e}^{\prime}}{E_{e}} \sin ^{2} \frac{\theta_{e}}{2}$.

For low $Q^{2}, y \approx 1-E_{e}^{\prime} / E_{e}$. Therefore, to reach high $y$ measuring at low $E_{e}^{\prime}$ is required.

Measurements at low $E_{e}^{\prime}$ are experimentally challenging. Dedicated triggers must be developed to collect the data with minimal bias at an acceptable trigger rate. The data are contaminated by the dominating photoproduction background for which $Q^{2} \sim 0$, and where the signature of the scattered electron is faked by a hadronic fi- 
nal state particle. The QED radiative corrections exceed $50 \%$.

The identification of the scattered electron uses combined calorimetric and tracking information. For H1, the electron $E_{e}^{\prime}$ and $\theta_{e}$ are measured using either the SpaCal $\left(12 \leq Q^{2} \leq 90 \mathrm{GeV}\right)$ or $\operatorname{LAr}\left(Q^{2} \geq 35 \mathrm{GeV}\right)$ calorimeter; for ZEUS the backward calorimeter is used. The minimal $E_{e}^{\prime}$ is set to $3.4 \mathrm{GeV}$ (H1 SpaCal), $3 \mathrm{GeV}$ (H1 LAr) and $6 \mathrm{GeV}$ (ZEUS). The electron trajectory is validated by the tracking detectors. A dedicated track reconstruction algorithm is developed by the ZEUS collaboration for this measurement to extend the angular coverage compared to the standard reconstruction.

In order to reduce background, cuts on the electron candidate shower shape variables and cluster-track matching are applied. Figure 2 shows an example of such electron identification variables for the H1 SpaCal analysis. The background is reduced by the electron identification cuts, nevertheless, it remains large and thus has to be estimated with high precision.
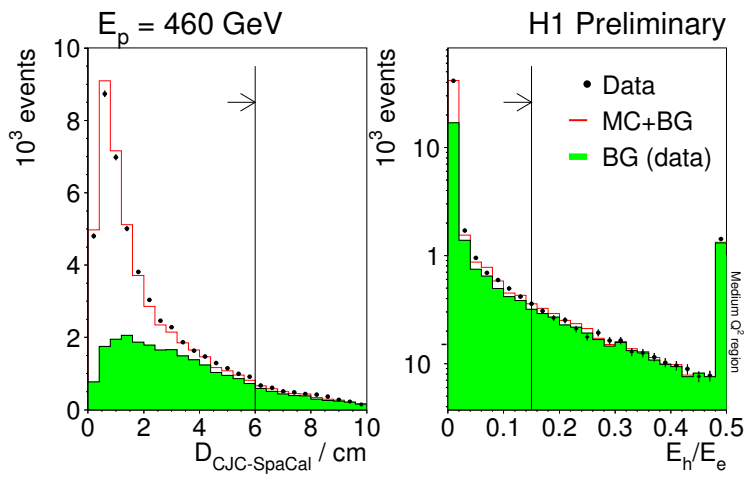

Figure 2. Electron identification variables used for H1 SpaCal analysis. The data are shown for the $E_{p}=460 \mathrm{GeV}$ run and for the high $y$ selection, $3.4 \leq E_{e}^{\prime} \leq 10 \mathrm{GeV}$. Left: transverse distance between the SpaCal cluster and the electron track projected to the SpaCal $z$ position. Right: ratio of the energy measured in the hadronic to the electromagnetic section of the SpaCal for the electron candidate clusters.
The ZEUS collaboration employs a Monte Carlo simulation (MC) techniques to estimate the photoproduction background using the PYTHIA [10] event generator. For about $15 \%$ of the photoproduction events the scattered electron is detected in a calorimeter located at $6 \mathrm{~m}$ from the nominal interaction point along the electron beam direction. These tagged events are used to normalise and check the background simulation. A similar detector is also used by the H1 collaboration for cross checks of the photoproduction background.

The H1 analysis estimates the background directly from data. Figure 3 shows the $E / p$ distribution for the H1 SpaCal analysis with the energy $E$ measured in the SpaCal and the momentum $p$ in the CJC. The sign of $p$ is taken as the parti-

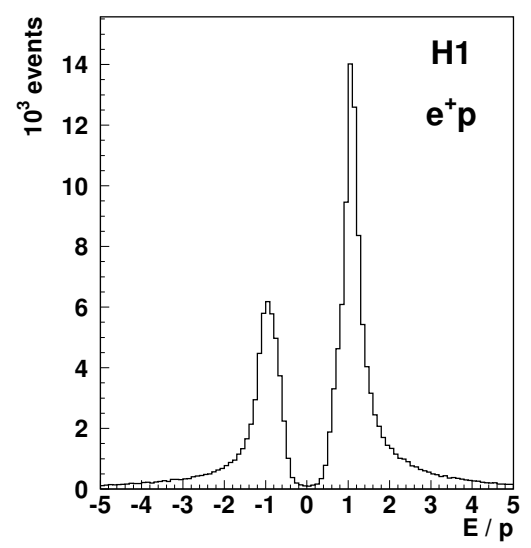

Figure 3. Distribution of energy over momentum for tracks linked to clusters in the SpaCal with $3.4 \leq E_{e}^{\prime} \leq 10 \mathrm{GeV}$. Tracks with a negative charge are assigned a negative $E / p$.

cle charge. For the scattered lepton the reconstructed charge is expected to coincide with the lepton beam charge (positive for the runs with reduced $E_{p}$ ). The sample with the charge opposite to the beam provides the background sample. Assuming charge symmetry of the background, 
this opposite charge sample should be subtracted from the correct charge sample to obtain the DIS signal. For application of this method, it is crucial to control the background charge asymmetry ratio $\kappa$, which may deviate from unity mainly because of differences in the reconstruction of opposite charge background particles in the detector. Using about equal size samples of $e^{+} p$ and $e^{-} p$ data collected at $E_{p}=920 \mathrm{GeV}$ with a total luminosity of $96 \mathrm{pb}^{-1}, \kappa$ is measured to $0.5 \%$ precision.

The leading order QED radiative effects are included in MC simulation using HERACLES [11] as implemented in the DJANGO [12] program. The corrections are checked using the HECTOR [13] program. Figure 4 shows an example of radiative corrections $\delta=\sigma_{\text {total }} / \sigma_{\text {born }}-1$ as a function of $y$. The total correction exceeds $50 \%$ for $y>0.7$. The largest component of the corrections is due to initial state radiation (ISR) which corresponds to the radiative return to $y \sim 0$ and smaller $Q^{2}$, where the cross section is large.

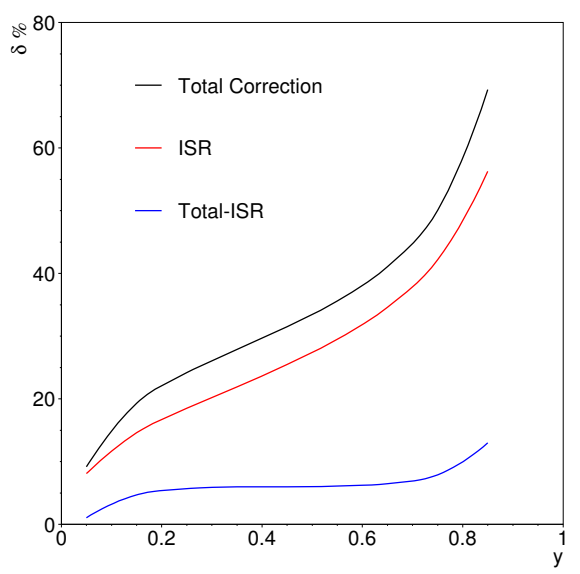

Figure 4. QED radiative corrections $\delta$ as a function of $y$ for $Q^{2}=25 \mathrm{GeV}^{2}$ estimated using HECTOR [13]. The top line corresponds to the total corrections, the middle line corresponds to contribution of the ISR, and the bottom line shows total minus ISR corrections.
The ISR contribution can be controlled using

$E-p_{Z}=\sum_{h}\left(E^{h}-p_{z}^{h}\right)+E_{e}^{\prime}\left(1-\cos \theta_{e}\right)$,

where the sum runs over all hadronic final state particles $h$, and $E^{h}$ and $p_{z}^{h}$ are energy and longitudinal momentum of each particle. The sum does not include the ISR photon which typically escapes along the beam line. From the energymomentum conservation, $E-p_{Z}$ is equal to twice the effective beam energy. Figure 5 shows comparisons of data and MC for the H1 SpaCal analysis after background subtraction. The bottom
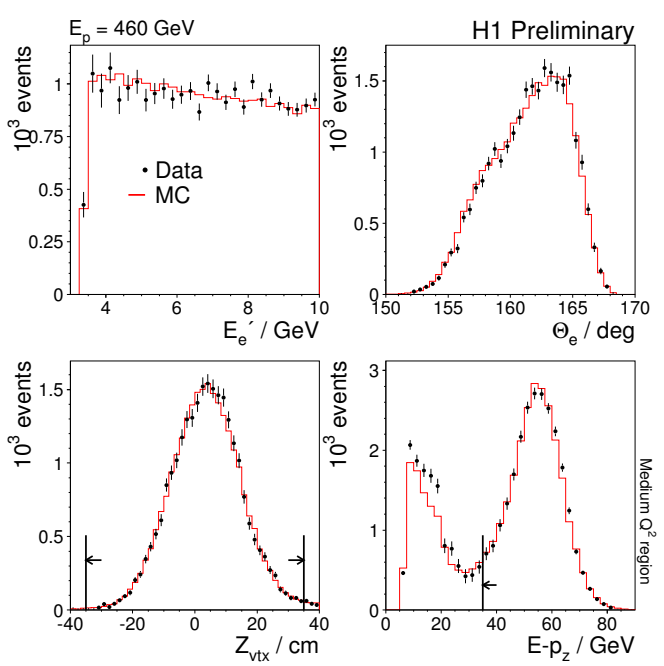

Figure 5. For the H1 SpaCal analysis, from top to bottom and left to right: distributions of $E_{e}^{\prime}$, $\theta_{e}$, the event vertex $Z_{\mathrm{vtx}}$ and $E-p_{Z}$ after background subtraction. The plots are shown for $3.4 \leq E_{e}^{\prime} \leq 10 \mathrm{GeV}$, with vertical lines indicating analysis cuts.

right plot shows the $E-p_{Z}$ distribution. Two peaks can be clearly separated in this distribution, one corresponding to $E-p_{Z} \sim 55 \mathrm{GeV}$, twice $E_{e}$, the other to $E-p_{Z}<20 \mathrm{GeV}$, twice $E_{e}^{\prime}$. The cut $E-p_{Z}>35 \mathrm{GeV}$ removes most of the ISR events. The radiative events at $E-p_{z}<$ 
$35 \mathrm{GeV}$ are described with an accuracy of $10 \%$. Since the remaining radiation does not exceed $10 \%$, the uncertainty induced by radiative corrections for the high $y$ cross section measurement is estimated to be less than $1 \%$.

Both $\mathrm{H} 1$ and ZEUS collaborations performed cross section measurements at high $y$ using large statistics samples collected at $E_{p}=920 \mathrm{GeV}$. For example, the $\mathrm{H} 1$ measurements for $y=0.825[14]$ are shown in figure 6 . Compared to the published

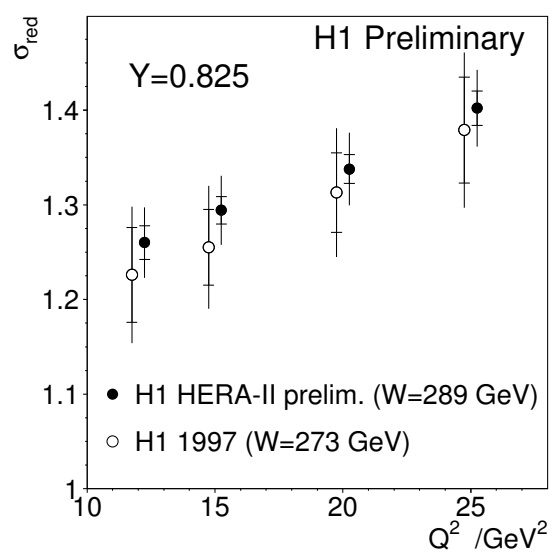

Figure 6. The reduced inclusive DIS cross section measured at $y=0.825$ and shown as a function of $Q^{2}$ for the published H1 data [15] taken at $E_{p}=820 \mathrm{GeV}$ (open circles) and the preliminary $\mathrm{H} 1$ data at $E_{p}=920 \mathrm{GeV}$ (full circles). The inner error bars show statistical and the outer error bars show statistical and systematic errors added in quadrature. The absolute luminosity uncertainty is not included in the error bars.

$\mathrm{H} 1$ result [15], the preliminary $\mathrm{H} 1$ measurement improves the statistical uncertainties by a factor of three and the total uncertainties by a factor of two.

The measurements of the reduced double differential cross section at the nominal and reduced proton beam energies by the H1 and ZEUS collaborations are shown in figure 7 and figure 8 , re- spectively. The data are compared to the H1 PDF 2000 [16] and ZEUS-JETS [17] parameterisations. For each $Q^{2}$ bin and at the lowest $x$, the cross

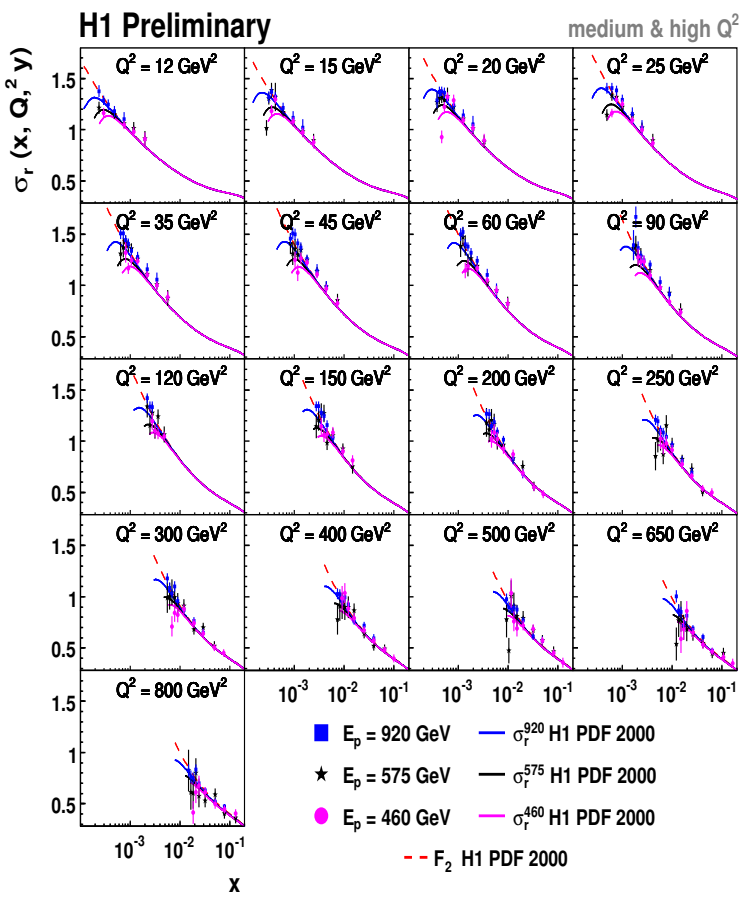

Figure 7. The reduced inclusive DIS cross section measured by the $\mathrm{H} 1$ collaboration at different $Q^{2}$ values and shown as a function of $x$ for the data taken at the three proton beam energies, $E_{p}=920 \mathrm{GeV}$ (squares), $575 \mathrm{GeV}$ (stars) and $460 \mathrm{GeV}$ (circles). The error bars show the statistical and systematic errors added in quadrature. The curves correspond to the H1 PDF 2000 parameterisation and are shown (from top to bottom) for $F_{2}$ and for $\sigma_{r}$ at $E_{p}=920,575$ and $460 \mathrm{GeV}$.

sections show characteristic flattening and turnover, different for different centre-of-mass energies. This behaviour is explained by the influence of the longitudinal structure function $F_{L}$. Within the uncertainties, the data agree well with the expectations. 


\section{ZEUS}

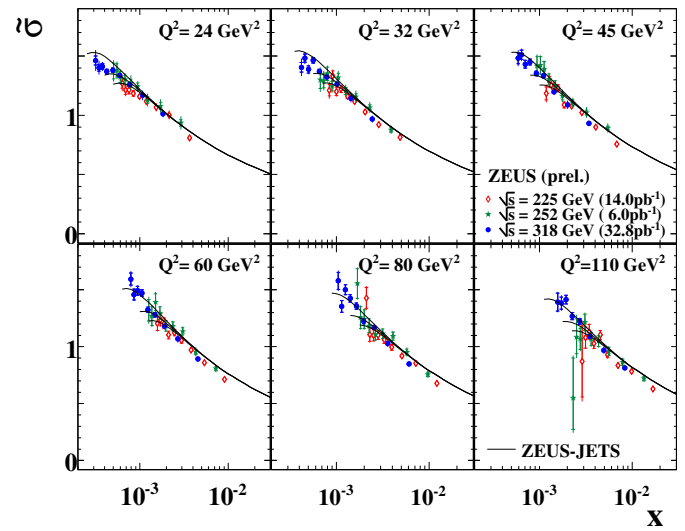

Figure 8. The reduced inclusive DIS cross section measured by the ZEUS collaboration at different $Q^{2}$ values and shown as a function of $x$ for the data taken at the three proton beam energies, $E_{p}=920 \mathrm{GeV}$ (full circles), $575 \mathrm{GeV}$ (stars) and $460 \mathrm{GeV}$ (open circles). The inner error bars show the statistical and the outer error bars show the statistical and systematic errors added in quadrature. The curves correspond to the ZEUS-JETS parameterisation.

\section{Measurement of $F_{L}$}

The structure function $F_{L}$ is determined using the reduced cross section measurements at the three proton beam energies. This procedure consists of two main steps and is illustrated in figure 9 .

A large uncertainty on $F_{L}$ stems from the relative normalisation of the data sets at different $E_{p}$. This uncertainty can be significantly reduced by demanding the measurements to agree at low $y$, where $f(y)$ is small, and the reduced cross section is almost equal to $F_{2}$. This regime is reached in figure 9 for the bins at $x=0.0016$ and $x=0.0025$. The uncertainty on the relative normalisation is then defined by the statistics at low $y$, a small residual dependence on $F_{L}$ and non-perfect cancellation of the systematic uncertainties. A rather large systematic error arises from the energy scale

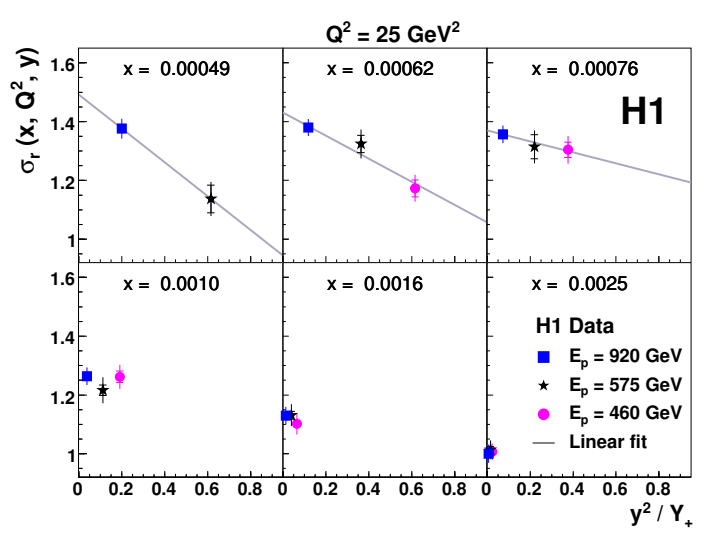

Figure 9. The reduced inclusive DIS cross section measured by the $\mathrm{H} 1$ collaboration and plotted as a function of $y^{2} / Y_{+}$for six values of $x$ at $Q^{2}=25 \mathrm{GeV}^{2}$ and $E_{p}=920,575$ and $460 \mathrm{GeV}$. The inner error bars denote the statistical error, the full error bars include the systematic errors. The luminosity uncertainty is not included in the errors. For the bins used to extract $F_{L}$, a straight line fit is shown where the slope determines $F_{L}$. The other bins are used for the relative normalisation of the data sets at different $E_{p}$.

uncertainty. The total uncertainty of the relative normalisation between different data sets is about $1.5 \%$.

The structure function $F_{L}$ is measured as a slope of a linear fit of $\sigma_{r}\left(x, Q^{2}, f(y)\right)$ versus $f(y)$. These fits are shown in figure 9 for the bins in $x$ at $0.00049,0.00062$ and 0.00076 . For the lowest $x$ bin the measurement with $E_{p}=460 \mathrm{GeV}$ is not possible, since it would lie beyond the kinematic limit $y=1$. Here $F_{L}$ is determined using data with $E_{p}=920$ and $575 \mathrm{GeV}$ only. For the other bins all three energies are used. The uncertainties on $F_{L}$ arise from statistics, from the uncertainty of the relative normalisation of the data sets and from the systematic uncertainties. Similar to the determination of the relative normalisation, there is a cancellation of the systematic uncertainties. However, this cancellation is not complete since 
the uncertainties typically increase at high $y$.

Figure 10 shows the first preliminary measurements of $F_{L}$ performed by the ZEUS collaboration [18]. The data are compared to the ZEUSJETS PDF parameterisation which is based on other ZEUS data. The data cover the range from $24 \leq Q^{2} \leq 110 \mathrm{GeV}^{2}$. A good agreement between the data and the expectation is observed.

\section{ZEUS}

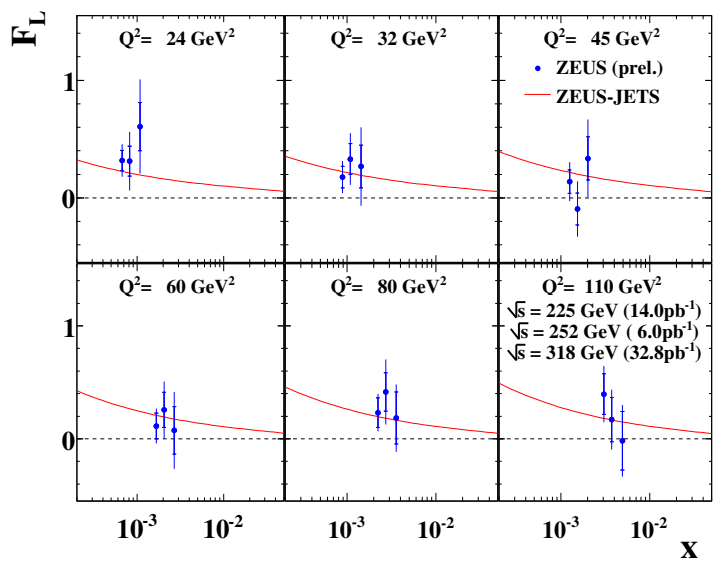

Figure 10. The longitudinal structure function $F_{L}\left(x, Q^{2}\right)$ measured by the ZEUS collaboration. The inner error bars denote the statistical error, the full error bars include the systematic errors. The curves represent the ZEUS-JETS PDF fit.

The measurements of $F_{L}$ obtained by the H1 collaboration [19] are shown in figure 11. This result is obtained using cross section data measured in the SpaCal and LAr calorimeters. The $F_{L}$ measurement based on the SpaCal data only is published in [20]; it is a subset of the results shown here. The SpaCal based measurements cover the range $12 \leq Q^{2} \leq 90 \mathrm{GeV}^{2}$, while the LAr data cover $35 \leq Q^{2} \leq 800 \mathrm{GeV}^{2}$. Use of the $\mathrm{LAr}$ data significantly improves the precision in the overlap region. The $\mathrm{H} 1$ measurements agree well with those obtained by the ZEUS collaboration.

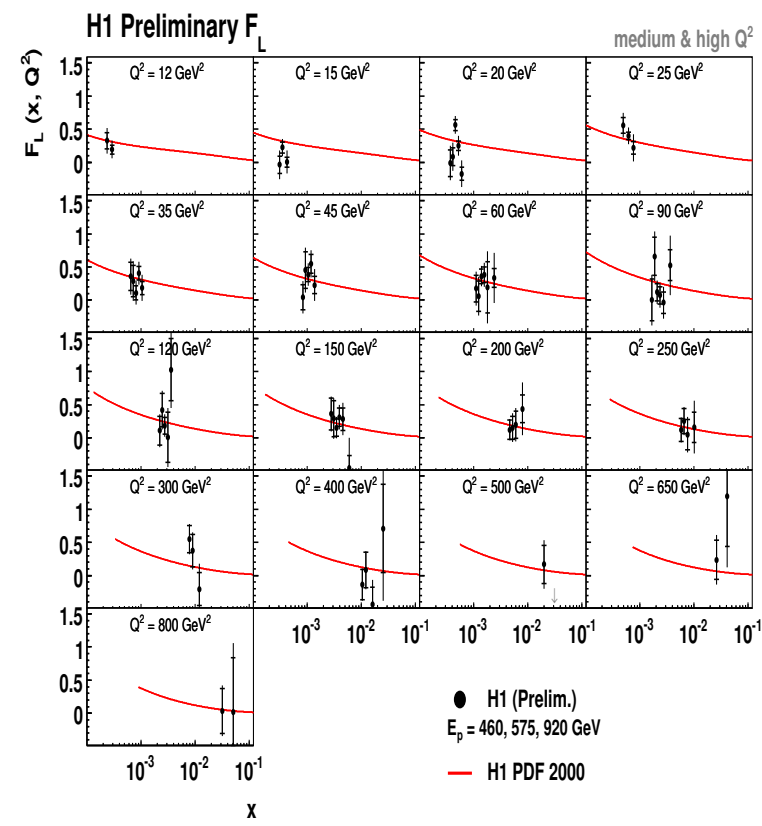

Figure 11. The longitudinal structure function $F_{L}\left(x, Q^{2}\right)$ measured by the $\mathrm{H} 1$ collaboration. The inner error bars denote the statistical error, the full error bars include the systematic errors. The curves represent the H1 PDF 2000 fit.

The measurements of $F_{L}$ performed by the H1 collaboration have large statistical uncertainties, which at low $Q^{2}$ stem from the background subtraction. The $x$ range covered by the measurements for each $Q^{2}$ bin is limited, and for this range the expected variation of $F_{L}$ is small. Therefore, the measurements are averaged for each $Q^{2}$ bin in order to obtain a more compact representation of the data. The averaged $F_{L}$ measurements are shown in figure 12 and are compared to various predictions. The models coincide at $Q^{2}>100 \mathrm{GeV}^{2}$, but start to diverge at low $Q^{2}$ due to differences in the gluon density. The data agree with all predictions within the experimental uncertainties. It is thus important to extend the measurement to low $Q^{2}$. This can be done using the backward silicon tracker. 


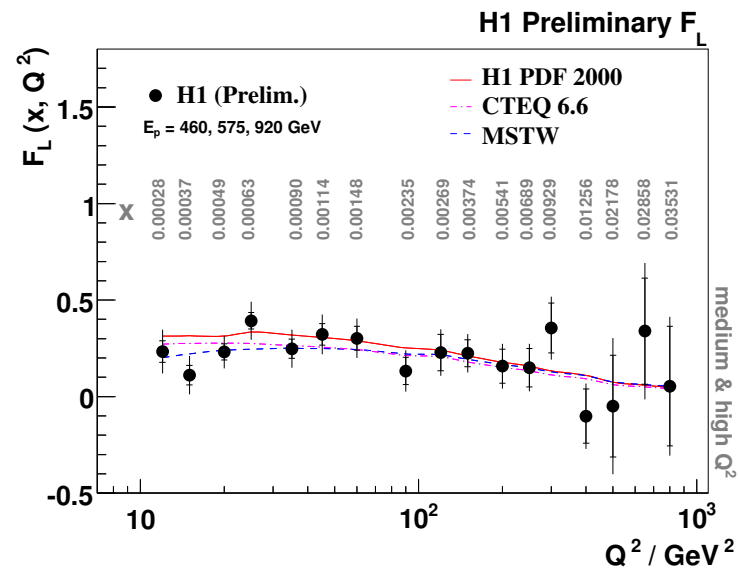

Figure 12. The H1 measurement of $F_{L}$ averaged over $x$ at fixed values of $Q^{2}$. The $x$ values of the averaged $F_{L}$ are shown above the data points. The theoretical predictions are calculated at the corresponding $x, Q^{2}$ points and connected by lines.

\section{Summary}

The H1 and ZEUS collaborations performed the first measurement of the longitudinal structure function $F_{L}$. The measurement is obtained using dedicated runs with reduced proton beam energies performed for the last three months of HERA operation. The experimental data agree well with each other and with the expectations based on the standard DGLAP evolution for which the parton densities are obtained from the DGLAP fits to the HERA reduced cross section data.

\section{REFERENCES}

1. E.D. Bloom et al., Phys. Rev. Lett. 23 (1969) 930.

2. D.J. Fox et al., Phys. Rev. Lett. 33 (1974) 1504.

3. J.K. Aubert et al. [EMC Collaboration], Phys. Lett. B 121 (1983) 87;

A.C. Benvenuti et al. [BCDMS Collaboration], Phys. Lett. B 223 (1989) 485;
L.W. Whitlow et al., Phys. Lett. B 250 (1990) 193 ;

M. Arneodo et al. [NMC Collaboration], Nucl. Phys. B 483 (1997) 3.

4. V.N. Gribov and L.N. Lipatov, Sov. J. Nucl. Phys. 15 (1972) 438;

V.N. Gribov and L.N. Lipatov, Sov. J. Nucl. Phys. 15 (1972) 675;

L.N. Lipatov, Sov. J. Nucl. Phys. 20 (1975) 94;

Y.L. Dokshitzer, Sov. Phys. JETP 46 (1977) 641;

G. Altarelli and G. Parisi, Nucl. Phys. B 126 (1977) 298.

5. C. Gwenlan, these proceedings.

6. E.A. Kuraev, L.N. Lipatov and V.S. Fadin, Sov. Phys. JETP 44 (1976) 443;

E.A. Kuraev, L.N. Lipatov and V.S. Fadin, Sov. Phys. JETP 45 (1977) 199;

I.I. Balitsky and L. N. Lipatov, Sov. J. Nucl. Phys. 28 (1978) 882.

7. M. Ciafaloni, Nucl. Phys. B 296 (1988) 49; S. Catani, F. Fiorani and G. Marchesini, Phys. Lett. B 234 (1990) 339;

S. Catani, F. Fiorani and G. Marchesini, Nucl. Phys. B 336 (1990) 18;

G. Marchesini, Nucl. Phys. B 445 (1995) 49.

8. L.V. Gribov, E.M. Levin and M.G. Ryskin, Phys. Rept. 100 (1983) 1;

A.H. Mueller, Nucl. Phys. B 268 (1986) 427; I. Balitsky, Nucl. Phys. B 463 (1996) 99;

V.V. Kovchegov, Phys. Rev. D 60 (1999) 034008;

M. Braun, Eur. Phys. J. C 16 (2000) 337;

E. Iancu, A. Leonidov and L.D. McLerran, Nucl. Phys. A 692 (2001) 583;

J. Bartels, L.N. Lipatov and G.P. Vacca, Nucl. Phys. B 706 (2005) 391;

J. Bartels and K. Kutak, Eur. Phys. J. C 53 (2008) 553.

9. R. Thorne, "The Longitudinal Structure Function at HERA", Proc. of XVI Int. Workshop on Deep-Inelastic Scattering and Related Topics, London, England, April 2008, http://dx.doi.org/10.3360/dis.2008.36.

10. T. Sjöstrand, Comp. Phys. Commun. 82 (1994) 74.

11. A. Kwiatkowski, H. Spiesberger and 
H.J. Moehring, Comp. Phys. Commun. 69 (1992) 155.

12. G.A. Schuler and H. Spiesberger, "Proc. of the Workshop on HERA Physics", vol.3 (1992) 1419, DESY, Hamburg.

13. A. Arbuzov et al., Comp. Phys. Commun. 94 (1996) 128.

14. N. Raicevic [for the H1 Collaboration], "Measurement of the Inclusive ep Scattering Cross Section at low $Q^{2}$ and high $y$ at HERA", Proc. of XV Int. Workshop on Deep-Inelastic Scattering, Munich, Germany, April 2007, http://dx.doi.org/10.3360/dis.2007.24.

15. C. Adloff et al. [H1 Collaboration], Eur. Phys. J. C 21 (2001) 33.

16. C. Adloff et al. [H1 Collaboration], Eur. Phys. J. C 30 (2003) 1.

17. S. Chekanov et al. [ZEUS Collaboration], Eur. Phys. J. C 42 (2005) 1.

18. D. Kollar [for the ZEUS Collaboration], "Measurement of the Longitudinal Structure Function $F_{L}$ at Low $x$ at HERA with the ZEUS Detector", Proc. of XVI Int. Workshop on Deep-Inelastic Scattering and Related Topics, London, England, April 2008, http://dx.doi.org/10.3360/dis.2008.37.

19. V. Chekelian [for the H1 Collaboration], "Direct $F_{L}$ Measurement at Hiqh $Q^{2}$ at HERA", Proc. of XVI Int. Workshop on Deep-Inelastic Scattering and Related Topics, London, England, April 2008,

http://dx.doi.org/10.3360/dis.2008.39.

20. F. Aaron et al. [H1 Collaboration], Phys. Lett. B 665 (2008) 139. 